\title{
The effect of rifampicin and isoniazid on liver and lung tissues in rats
}

\author{
Dalal F. Ahmed \\ Department of Biology/University of Mosul, Iraq \\ $\frac{\text { Received }}{1 . r .2011} \quad \frac{\text { Accepted }}{\mid 9.7 .2011}$
}

\section{ABSTRACT}

Objectives: The purpose of this study was to investigate the histopathological changes in Swiss albino rats liver and lung tissues resulted from the oral administration of the antituberculous drugs rifampicin and isoniazid.

Materials and Methods: The study was conducted on 24 adult rats aged 2.5-3 months weighing 200-250 g randomly distributed into four groups(6 animals for each), the first group served as a control group while the remaining three as test groups. The rifampicin group was treated with $50 \mathrm{mg} / \mathrm{kg} \mathrm{B.W}$. once per day for 60 days, the isoniazid group was treated with $25 \mathrm{mg} / \mathrm{kg} \mathrm{B}$.W. once per day for 60 days, the rifampicin and isoniazid groups were treated with $50,25 \mathrm{mg} / \mathrm{kg}$ B.W. respectively once per day for 60 days.

Results: Giving rifampicin alone orally once daily for 60 days caused fatty degenerative and necrotic changes in the liver in addition to proliferative lesions and emphysema in the lung. Rats receiving isoniazid alone orally once daily for 60 days showed similar degenerative and necrotic changes in liver and lung but of lower intensity. Whereas those receiving rifampicin and isoniazid combination for 60 days showed pathological changes similar to those induced when each of the two types of antituberculosis drugs was given alone but of more intensity.

Conclusion: Rifampicin and isoniazid showed clear histopathological changes in the rat's liver and lung tissues when given separately ; however, changes where more intense when givenin combination.

Keywords: Rifampicin, isoniazid, hepatotoxicity, emphysema.




Tuberculosis is one of the most widespread diseases and causes of death in the world.Tuberculosis is caused by Mycobacterium tuberculosis, which is relatively resistant to many antibiotics. As such, recovery from this disease may be long $^{1,2}$.

Anti-tuberculosis agents

containing two different compounds were used for the treatment of this disease, as well as long-term treatment in order to prevent the infection from occurring again ${ }^{3,4}$. The antituberculosis agents were found to have serious adverse effects such as hepatotoxicity, hepatitis and lung inflammation. Moreover, the damages caused by the intensity of the drug vary from simple diseases to more complex ones, such as liver failure. Two compounds, rifampicin and isoniazid, as well as ethanobutane and streptomycin- were used to cure the disease ${ }^{5,6}$.

Rifampicin is one of the semiartificial antibiotics which are derived from the Streptomycin group, and is used against Staphyllococcus, Mycobacterium and Brucella ${ }^{7}$. Rifampicin was found to lower the growth of most positive and negative bacteria to Gram pigment. Highest concentration of rifampicin in plasma is reached after 2 hours from oral treatment, and is distributed in significant concentrations throughout body fluids ${ }^{8}$. Administrating rifampicin yields various side effects including the appearance of red and orange colors in urine and saliva in addition to various secretions of the respiratory system, skin rash, vomiting and nausea, and when used with other compounds; liver and renal failure ${ }^{9}$.

Isoniazid, is used for the treatment of tuberculosis because it spreads throughout body fluids and cells when administered orally or by injection. Isoniazid acts as a prodrug which changes into its active form that inhibits the function of the enzyme responsible for the formation of the cell wall of the bacteria causing tuberculosis. However, studies found that isoniazid causes many side effects including, skin rash, fever, jaundice, back pain, infection and atrophy of optic nerve, and negative impact on the peripheral nerves and the central nervous system ${ }^{10}$.

Damage to the liver in various degrees could be the cause of administrating both rifampicin and isoniazid $^{11,12}$, but the question here is; do length of treatment period has an impact on liver and other body organs such as the lungs? The current study was designed to answer this question.

\section{Materials and Methods}

The study was conducted at the animalhouse in the College of Veterinary Medicine, University of Mosul from February-June 2010. Twenty-four adult Albino rats, aged 2.5-3 months and weighed 200-250 g were bred in a room with conditions suitable for this experiment.

\section{Experiment Plan}

Twenty-four adult rats were randomly and equally distributed into four groups. These groups were treated using Gavage needle for 60 days, as follows:

1- Control group: treated with $0.5 \mathrm{~mL}$ of distilled water.

2- Rifampicin group: treated with 50 $\mathrm{mg} / \mathrm{kg} \mathrm{B.W}$. of rifampicin once per day.

3- Isoniazid group: treated with 25 $\mathrm{mg} / \mathrm{kg} \mathrm{B}$.W. of isoniazid once per day.

4- Rifampicin and isoniazid group: treated with $50 \mathrm{mg} / \mathrm{kg} \mathrm{B}$.W., $25 \mathrm{mg} / \mathrm{kg}$ B.W.once per day, respectively.

At the end of the treatment stage, the rats were dissected to extract the livers and lungs after sedating the animals with ether. An abdominal incision was 
made, and then a scissor was inserted along the abdominal area till the sternum and the neck, after which the lungs and the liver were extracted and put in formalin for tissue sectioning ${ }^{13}$.

\section{Preparing Microscopic Slides}

The slides were prepared depending on Dury's method ${ }^{14}$, in which the slides are washed, dehydrated, cleared, infiltrated and embedded to get wax molds. Then, the slides were cut and spread to get tissue sections of 5-6 $\mu$ thickness, and spread in a hot water bath with $40-45^{\circ} \mathrm{C}$ temperature. The slides were then loaded on clean, labeled glass slides after making light scans of albumin. The slides then were left to dry at lab room temperature. At the end of the process, the tissue sections were tinted with HarsHematoxiline-eosin tincture according to Luna method (1968), and was loaded using Desterine Plasters-Zylene (DPX) and a glass lid, and were put on a hot plate with temperature of $4^{\circ} \mathrm{C}$ to hasten the dryness process.

\section{Test and Microscopic Photography}

The microscopic test of tissue sections was conducted using Compound photo microscope. Microscopic photos of tissue sections were taken using BElPhotonice microscope.

\section{Results \\ Liver tissue morphology without treatment}

The liver is one of the supplements of the digestive system, and one of the largest glands of the human body. It is surrounded by a capsule, which divides the liver into several lobules. At the center of each lobule lies the central vein. The mater of the liver is formed of long, irregular and branching cells, which extend from the central vein to the lobe outline layer. The hepatic cells are distinguished for its polygonal shape, and having one central nucleus and acidic cytoplasm. Spaces within the hepatic plates are called sinusoids, which open up in the central vein. The hepatic artery, portal vein, bile duct and the lymphatic vessels are located at the intercellular septums (figure1).



Figure1. The central vein (A), hepatic plates (B) and hepatic sinusoids (C) in the control rat (300X) 


\section{Tissue changes resulting from rifampicin treatment on liver}

Lab tests showed hepatic coagulative necrosis, congestion of the blood



Figure 2: Coagulative necrosis of hepatic cells (A) and congestion of the blood vessel (B) and fatty degenerative (C) in the rifampicin treated rat $(1000 \mathrm{X})$

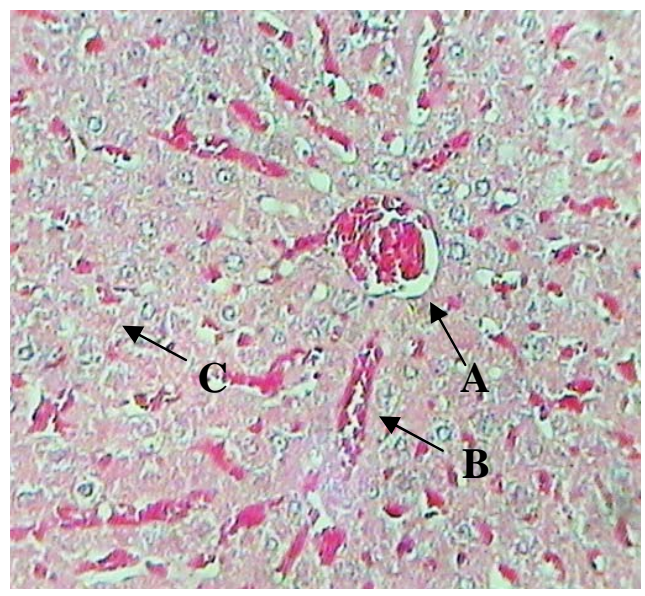

Figure 4: Congestion of central vein(A), liver sinusoids(B) and liver necrosis (C) in the rifampicin treated rat $(400 \mathrm{X})$ vessels (Figure 2), the central vein (Figure 3), hepatic sinusoids (Figure 4) and interlobular septums (Figure5).

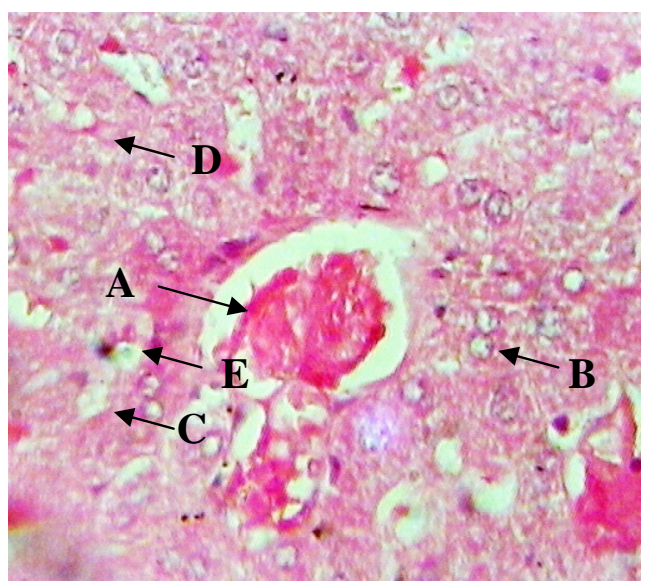

Figure 3: Congestion of central vein(A), numerous sites locations ofcoagulative necrosis $(B, C, D)$, fatty degenerative $(E)$ in the rifampicin treated rat $(1000 \mathrm{X})$

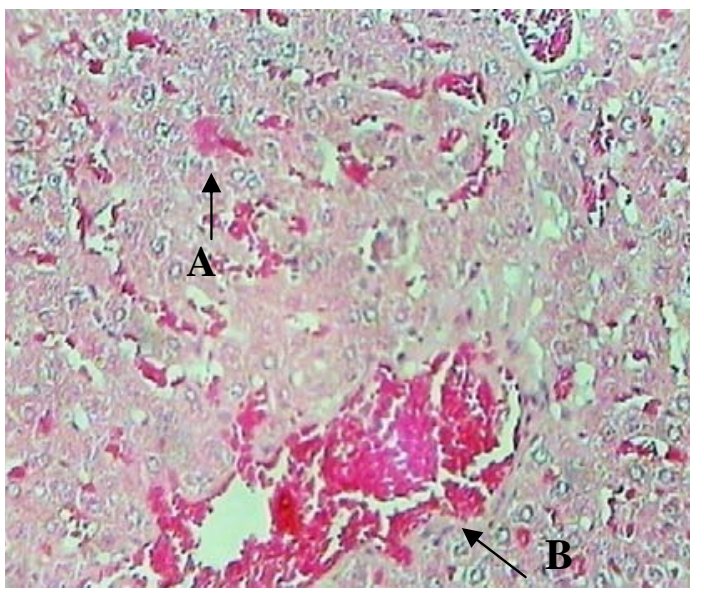

Figure 5: Congestion of liver sinusoid (A) and the interlobular septums(B) (400X) in the rifampicin treated rat 


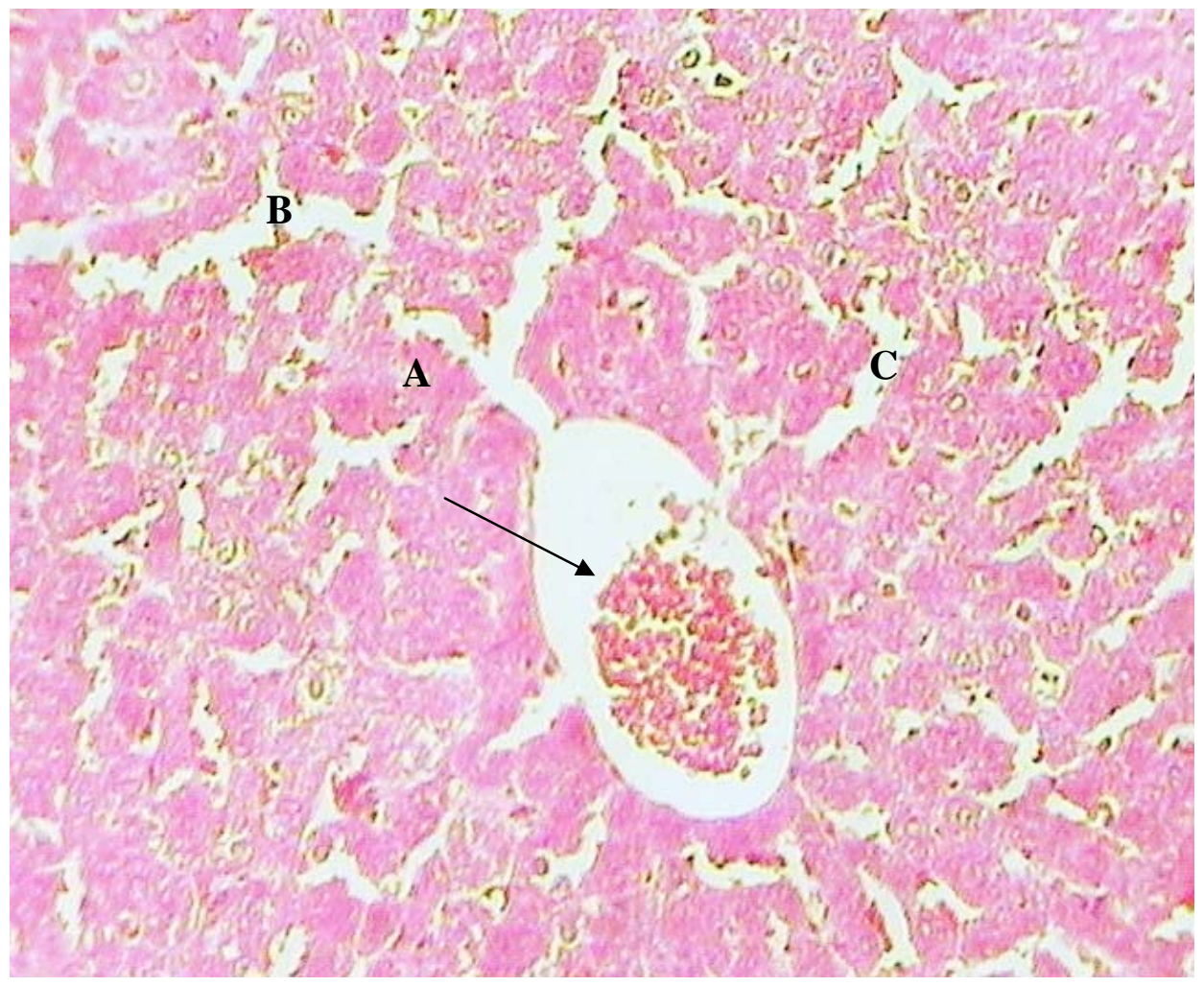

Figure 6: Congestion of central vein (A) and the overall change in liver tissue $(B, C)$ In the isoniazide treated rat

Tissue changes resulting from isoniazid treatment on liver

Congestion of the central vein with overall change in hepatic tissue morphology (Figure 6).

Tissue changes resulting from rifampicin and isoniazid treatment on liver

Severe congestion of the interlobular septum with severe infiltration of inflammatory cells (figure 7). Moreover, severe congestion in the central vein, hepatic coagulative necrosis and congestion of hepatic sinusoids and fatty degenerative are also seen (figure 8), which lead to alteration in liver tissue morphology (figure 9). Infiltration of portal area and hepatic portal vein are also seen (figure 10). 


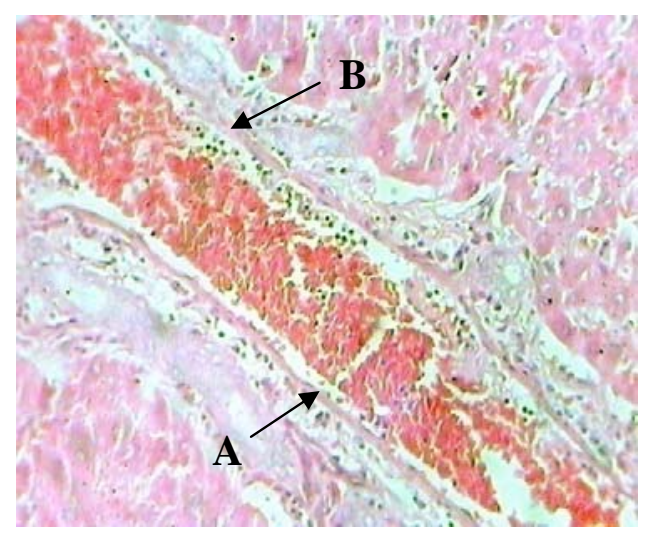

Figure 7: Severe congestion in the interlobular septums (A), and infiltration of sever inflammatory cells (B) in the rifampicin and isoniazide treated rat (1000X)

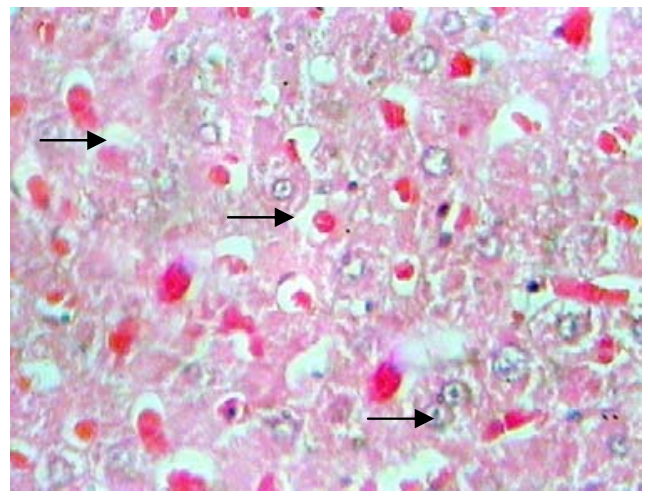

Figure 9: Overall change in liver morphology in the rifampicin and isoniazide treated $\operatorname{rat}(400 \mathrm{X})$



Figure 8: Congestion of the central vein, (A) coagulative necrosis of hepatic cells (B) and congestion of hepatic sinusoids

(C) and fatty degenerative (D) in the rifampicin and isoniazide treated $\operatorname{rat}(1000 \mathrm{X})$



Figure 10: Slight infiltration in the inflammatory cells, (A) congestion of the portal vein (B) and infiltration of the portal area $(C)$ in the rifampicin and isoniazide treated rat $(400 \mathrm{X})$

pleura filling the thoracic cavity. The trachea branches into two primary bronchi, which in turn branch to smaller bronchi and end up in the terminal bronchioles and alveoli. (figure11). 


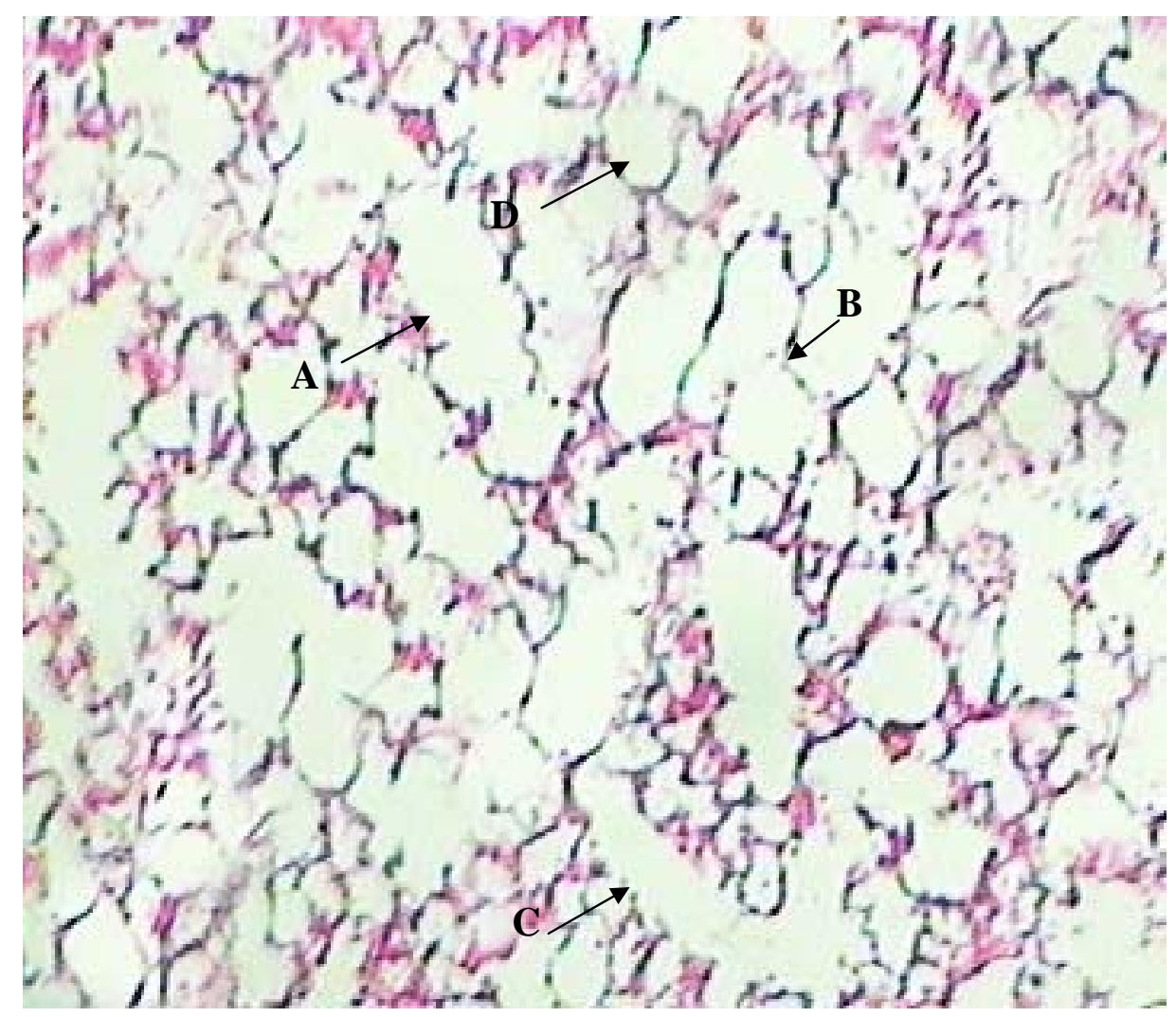

Figure 11: Overall composition of the normal lung tissue; alveolar duct (A), alveolarsac (B) and pulmonary duct (C) and pulmonary alveoli are visible (D) (400X)

\section{Changes in lung tissue caused by rifampicin effect}

Microscopic test clearly showed emphysema and infiltration of inflammatory cells between the alveoli
(Figures 12, 13), congestion around the bronchioles and inside the alveoli

(Figure 14), and infiltration of inflammatory cells inside and outside of the pulmonary alveoli (Figure 15). 




Figure 12. Emphysema in the rifampicin treated rat $(1000 \mathrm{X})$

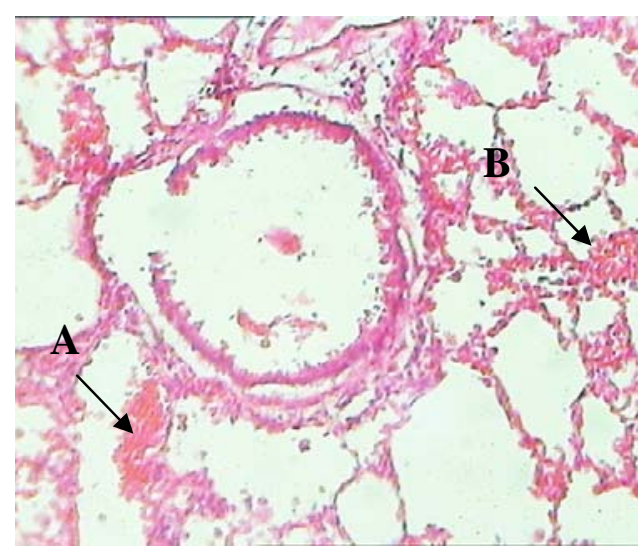

Figure14. Bloody congestion around the bronchiole (A) and the pulmonaryalveoli (B) in the rifampicin treated rat $(1000 \mathrm{X})$

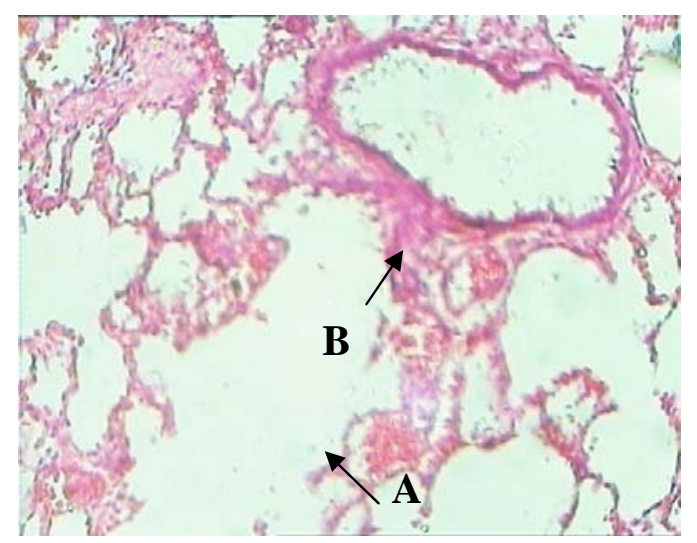

Figure 13. Emphysema (A) and bleeding around the bronchiole(B) in the rifampicin treated rat $(1000 \mathrm{X})$



Figure 15. Infiltration of inflammatory cells inside (A) and outside of the pulmonaryalveoli(B) in the rifampicin treated rat $(1000 \mathrm{X})$

between alveoli, (Figure16) in addition to emphysema occurred (Figure 17). 




Figure 16. Infiltration of inflammatory cells inside (A) and outside (B) of the alveoliand around the terminal bronchioles $(\mathrm{C})$ in the isoniazid treated rat $(1000 \mathrm{x})$

\section{Changes in lung tissue caused by} rifampicin and isoniazid

Severe alterations around the terminal bronchioles, namely, infiltration of inflammatory cells were found (figures
$18,22)$, in addition to emphysema (figures 19, 20, 21) and severe infiltration of inflammatory cells inside and outside of the alveoli (figure 20).

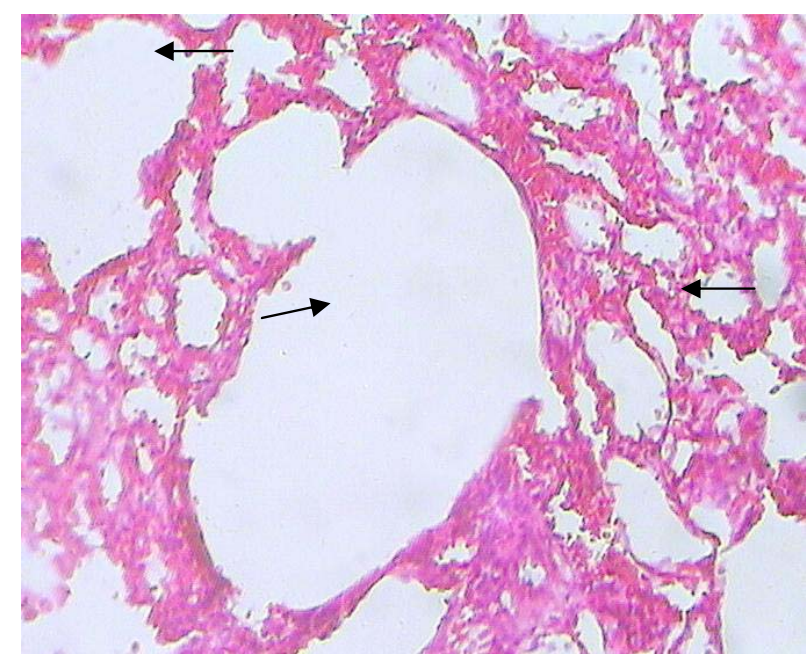

Figure 17. Emphysema in the isoniazid treated rat(1000X) 




Figure 18. Sever Infiltration of inflammatory cells around the bronchioles in the rifampicin and isoniazid teated rat (1000X)

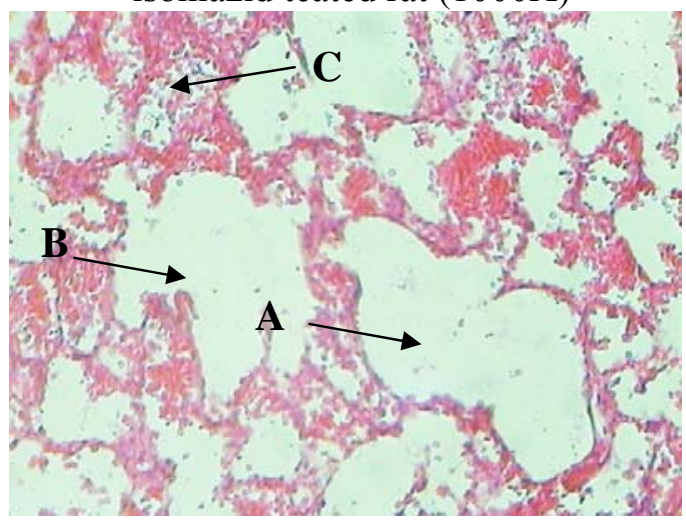

Figure 20. Spreading of emphysema(A) and infiltration of inflammatory cells (C) in the rifampicin and isoniazid teated rat



Figure 19. Spreading of emphysema in the rifampicin and isoniazid teated rat (1000X)



Figure 21. Spreading of emphysema in the rifampicin and isoniazid teated rat (400X)

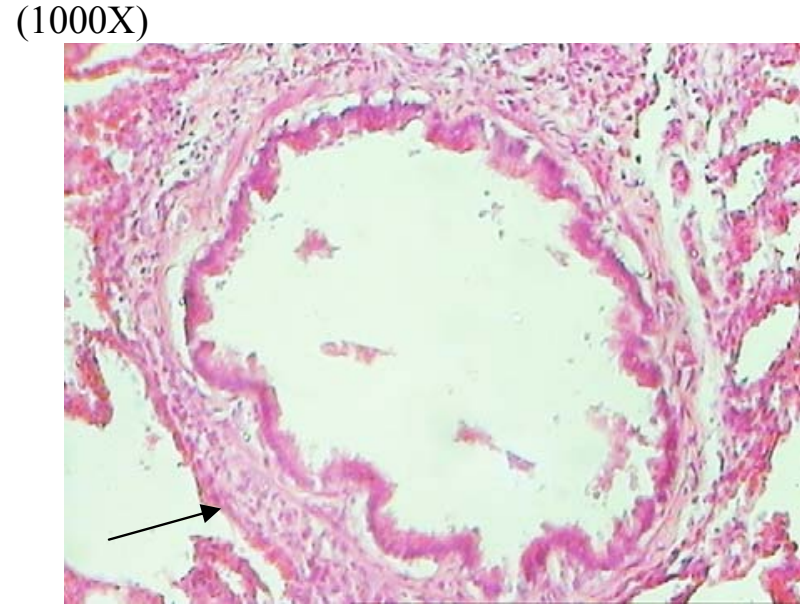

Figure 22. Severe infiltration of inflammatory cells around the bronchiole in therifampicin and isoniazid teated rat (1000X) 


\section{Discussion}

Drug-induced liver disease is a wellknown side effect of several drugs that are used for the treatment of active tuberculosis or latent tuberculosis infection. The duration of treatment with isoniazid is 9 months. This long duration might increase the risk of hepatotoxicityand infection with isoniazid- resistant organisms, therefore, a shorter 3 month regimen of isoniazid plus rifampicin regimen has been recommended by the British Thoracic Society ${ }^{1}$.

The present study showed several changes in the form of congestion, necrosis and degenerative changes, compared to the control group. This is in line withEna $\mathrm{J}$ findings in 2005, who recorded $2.6 \%$ hepatoxicity when administrating rifampicin and isoniazid simultaneously, compared with $1.1 \%$ hepatoxicity when administrating rifampicin alone, and $1.6 \%$ when administrating isoniazid alone to patients with tuberculosis ${ }^{15}$. These findings are not clearly explained, however,several proposed mechanisms have been suggested which include dose-related toxicity, oxidative stress, lipid peroxidation, immune-related, induction of liver enzyme in the hydrolase system, thus enhancing the toxicity of some of the isoniazid toxic metabolites, activation of CYP2E1, and reduced glutathione level ${ }^{16,17}$.

$$
\text { Hepatoxicity caused by }
$$

rifampicin could also explained by hyperbilirubinemia, which overlaps with the secretion of bilirubin, consequently causing chloestasis, increased fat oxides and difference in liver transaminase enzymes ${ }^{15}$.
Administrating rifampicin for long periods of time may lead to death as a result of waxing and liver cirrhosis ${ }^{18}$.

In the course of the current study, it was impossible to draw conclusion as what drug has the most impact on the liver. As such, the idea of rifampicin increases the risk of liver damage when administered with isoniazid $^{15}$, but in light of the results drawn from the present study, administrating rifampicin alone caused more damage on the liver than administrating isoniazid alone, which suggests the risk of using rifampicin on the long-term.

The study also showed the occurrence of various side effects on lung tissue in all three groups (groups 2,3 and 4), which include lung swelling caused by free roots affecting lung tissue, consequently leading to rupture of pulmonary alveoli, and consequently, emphysema, in addition to infiltration of inflammatory cells inside and outside of the pulmonary alveoli and inflammation of pulmonary bronchioles but with varying degrees, as the isoniazid and rifampicin treated group showed more alterations than in the group treated with the two drugs separately ${ }^{19}$.

In conclusion,rifampicin given alone has shown a greater effect on the liver than isoniazid given alone suggesting a greater role of rifampicin in the drug induced hepatotoxicity. However, combined drug administration (rifampicin and isoniazid) revealed more intense effects. 


\section{References}

1. Goodman and Gilman S. Manual of pharmacology and therapeutics $11^{\text {th }}$ ed. NY: McGraw-Hill companies; 2008.

2. Nuermberger E, Bishai WR, Grosset JH. Latent tuberculosis infection. Semin Respir Crit Care Med 2004;25:317-36.

3. Leibert W, Rom WW. Principles of tuberculosis management of AIDS related opportunistic infections $10^{\text {th }}$ ed Philadelphia: Lippincott, Williams \& Wilkins; 2004 pp.713728.

4. Bass JB, Farer LS, Hopwell PC. Treatment of tuberculosis and tuberculosis infections in adult and children. ATS and Centers for Disease Control and Prevention. Am J Respir Crit Care Med 1994;149:1359-1374.

5. Menzies D, Long R, TrajmanA, et al. Adverse events with 4 months of Rifampin Therapy or 9 Months of Isoniazid Therapy for Latent tuberculosis infection. Ann Intern Med 2008;149:689-97

6. Peter VB, MellisJB ." clinical pharmacology". $10^{\text {th }}$ edLondon:2008.p.221.

7. Farr BF. Rifampicins. In: Mandell D, Bennett S. Principles and infectionsdiseases $\quad 5^{\text {th }}$ ed.Philadelphia:Churchill Livingstone; 2000. P. 348-361.

8. Grosset J, Leventis S. Adverse effects of rifampin. Rev Infect Dis 1983;5: S440-S446.

9. Barners PF, Barrows SA. Tuberculosis in the 1990s. Ann Inter Med 1993;119: 400-410.

10. Chong S, Hongzi Z, Guoliang Z. Isoniazid-induced hepatotoxicity in rat hepatocytes of gel entrapment culture. Toxicology letters2006;167(1):66-74.
11. Fahmi YK, Fatima R. Rifampicinisoniazid induced fatal fulminant hepatitis during treatment of latent tuberculosis: A case report and literature review. Indian J Crit Care Med 2010;14:97-100.

12. Gangadharam PR. Isoniazid, rifampicin, and hepatotoxicity. Am Rev Respir Dis 1986;133:963-965.

13. Dury RAB, Wallgton EA, Cameron, SR. Carleton's histological techniques. $4^{\text {th }}$ edition. New York: Oxford University Press; 1985.

14. Luna, LG. Manual of histological staining methods of the armed forces $3^{\text {rd }}$ ed. New York: McGrawHill book company; 1968.

15. Ena J, Valls V. Short-course therapy with rifampicin plus isoniazid, compared with standard therapy with isoniazid, for latent tuberculosis infection: a metaanalysis. Clin Infect Dis 2005;40:670-6.

16. Kalor BS, Aggarwal S, Khurana, et al. Effect of cimetidine on hepatotoxicity induced by isoniazid-rifampicin combination in rabbits. Indian $\mathrm{J}$ Gastroenterol 2007;26:18-21.

17. Attri S, Rana SV, Vaiphei K, et al. Isoniazid and rifampicin-induced oxidative hepatic injury--protection by $\mathrm{N}$ acetylcysteine. Hum Exp Toxicol 2000;19:517-522.

18. Tostmann A, Boeree MJ, Aarnoutse R, et al. Antituberculosis drug-induced hepatotxicity: Concise up-to-date review. J Gastroenterol and Hepatol 2008; 23:192-202.

19. McCoenin MD, Zachary JF. Pathological basis veterinary disease. $4^{\text {th }}$ edition Mosby: Elsevier Inc; 2007. p.495-496. 